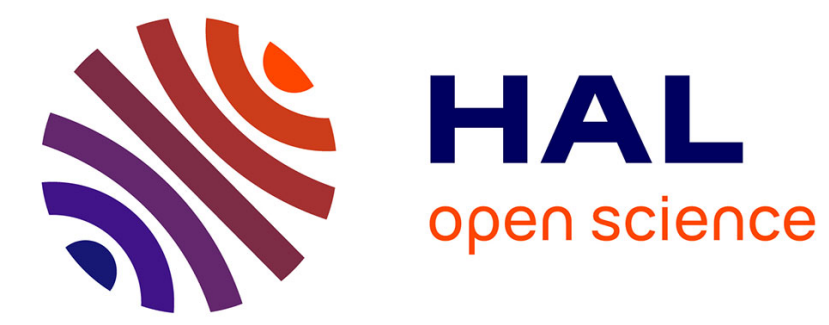

\title{
AC Stark-shift in CPT-based Cs miniature atomic clocks
}

\author{
D. Miletic, C. Affolderbach, M. Hasegawa, R. Boudot, C. Gorecki, G. Mileti
}

\section{To cite this version:}

D. Miletic, C. Affolderbach, M. Hasegawa, R. Boudot, C. Gorecki, et al.. AC Stark-shift in CPTbased Cs miniature atomic clocks. Applied Physics B - Laser and Optics, 2012, 109, pp.89-97. 10.1007/s00340-012-5121-7 . hal-00734965

\section{HAL Id: hal-00734965 \\ https://hal.science/hal-00734965}

Submitted on 15 May 2021

HAL is a multi-disciplinary open access archive for the deposit and dissemination of scientific research documents, whether they are published or not. The documents may come from teaching and research institutions in France or abroad, or from public or private research centers.
L'archive ouverte pluridisciplinaire HAL, est destinée au dépôt et à la diffusion de documents scientifiques de niveau recherche, publiés ou non, émanant des établissements d'enseignement et de recherche français ou étrangers, des laboratoires publics ou privés. 


\title{
AC Stark-shift in CPT-based Cs miniature atomic clocks
}

\author{
D. Miletic, C. Affolderbach, M. Hasegawa, R. Boudot, C. Gorecki, G. Mileti
}

\begin{abstract}
We report on studies on the light-shift in caesium miniature atomic clocks based on coherent population trapping (CPT) using a micro-fabricated buffer-gas cell (MEMS cell). The CPT signal is observed on the Cs D1-line by coupling the two hyperfine ground-state Zeeman sublevels involved in the clock transition to a common excited state, using two coherent electromagnetic fields. These light fields are created with a distributed feedback laser and an electro-optical modulator. We study the light-shift phenomena at different cell temperatures and laser wavelengths around $894.6 \mathrm{~nm}$. By adjusting the cell temperature, conditions are identified where a miniature CPT atomic clock can be operated with simultaneously low temperature coefficient and suppressed light-shift. The impact of the light-shift on the clock frequency stability is evaluated. These results are relevant for improving the long-term frequency stability of CPT-based Cs vapour-cell clocks.
\end{abstract}

\section{Introduction}

Coherent population trapping (CPT) can be observed, for example, on the alkali D-lines by coupling two ground-state atomic levels to a common excited state, using two coherent

D. Miletic, C. Affolderbach, G. Mileti Laboratoire Temps-Fréquence (LTF), Institut de Physique, Université de Neuchâtel, Avenue de Bellevaux 51,

2000 Neuchâtel, Switzerland

e-mail: danijela.miletic@unine.ch

G. Mileti

e-mail: gaetano.mileti@unine.ch

M. Hasegawa $\cdot$ R. Boudot $\cdot$ C. Gorecki

FEMTO-ST, CNRS et Université de Franche-Comté,

32 avenue de l'observatoire, 25044 Besançon cedax, France electromagnetic fields in a so-called $\Lambda$-scheme. When the Raman resonance condition is satisfied, the atoms are trapped in a coherent superposition of the ground states (the dark state) where, in the ideal case, they do not absorb incident photons anymore. CPT resonances have been studied for 40 years: Alzetta et al. [1] observed dark lines in a sodium cell and already Bell et al. [2] reported a similar phenomenon at the Zeeman frequency of $\mathrm{Cs}$ and $\mathrm{Rb}$ vapours using an amplitude-modulated spectral lamp. The phenomenon was explained theoretically by an interference effect that keeps atoms in the non-absorbing state [3,4]. The simple model of a three-level system shows good agreement with the experimental results obtained on alkali atoms [5-7], in spite of their more complicated atomic structure. The phenomenon of CPT and its related effect, electromagnetically induced transparency (EIT) [8-10] is used in several applications such as magnetometry [11-13], laser cooling of atoms [14], and slow light $[15,16]$. The possibility to obtain narrow CPT resonances $[17,18]$ led to their application in atomic frequency standards ("atomic clocks") [19], where the CPT resonance is used as atomic reference to which the frequency of a quartz oscillator is stabilized [20-22]. In this case the $\Lambda$ scheme is formed by the $6 \mathrm{~S}_{1 / 2}$ ground-state components $\mid F=3$, $m_{\mathrm{F}}=0>$ and $\mid F=4, m_{\mathrm{F}}=0>$ involved in the "clock transition" of atomic Cs [19] and one of the hyperfine-split excited states $\left(6 \mathrm{P}_{1 / 2}, F^{\prime}=3\right.$ or 4 in our case), see Fig. 1 . Unlike optically pumped double-resonance atomic clocks, CPT-based atomic clocks do not require a microwave cavity, allowing a strong miniaturization of the device [23-26].

\section{Light-shift in coherent population trapping}

Sources of atomic clock instability at intermediate- to longterm time scales include the frequency shifts of the clock 


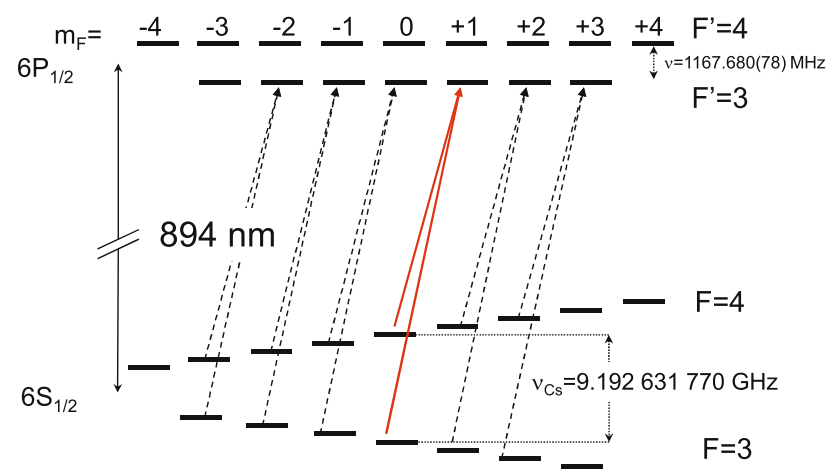

Fig. 1 Energy scheme of the Cs atom for the levels involved in the Cs D1 line. Coupling of the $F=3$ and $F=4$ ground states via the $F^{\prime}=3$ excited state is shown using circularly polarized light (dashed black arrows). Solid red arrows correspond to the coupling of the ground states with $m_{\mathrm{F}}=0$ to a common excited state $F^{\prime}=3$, $m_{\mathrm{F}}=1$, used as frequency reference in the studies reported here. Zeeman splitting of the excited state is not indicated, being not relevant here. The unperturbed hyperfine ground-state splitting frequency in $\mathrm{Cs}$ is $v_{\mathrm{Cs}}=9.192631770 \mathrm{GHz}$

resonance due to residual variations of, e.g., the external magnetic field, the buffer-gas pressure in the resonance cell, the temperature of the clock cell, the properties of the laser light (through the light-shift effect), and others $[22,27]$. It is therefore critical to carefully stabilize all these parameters and to find a clock operational scheme that minimizes the sensitivity of the clock frequency towards them. The light-shift is well known as one of the main sources of instabilities in CPT-based [28] and other atomic clocks.

The light-shift (AC Stark-shift) is the shift of atomic energy levels due to the interaction of the induced atomic dipole moment with the oscillating electric field of the light [29, 30]. The light-shift $\delta v_{i}$ of an atomic level $i$ can be approximated by a sum of dispersive Lorentzians (as a function of a single laser frequency $f_{\mathrm{L}}$ and intensity $\left.I \sim\left|\Omega_{\mathrm{R}}\right|^{2}\right)$ ) of width $\Gamma_{i k}$ centred at the optical atomic transition frequency $f_{i k}$, [27, 31]:

$\delta v_{i}\left(f_{\mathrm{L}}, I\right)=\frac{1}{4} \sum_{k=3}^{4}\left|\Omega_{R k}\right|^{2} \frac{\left(f_{\mathrm{L}}-f_{i k}\right)}{\left(f_{\mathrm{L}}-f_{i k}\right)^{2}+\Gamma_{i k}^{2} / 4}$

In the case of interest here, the optical transitions at $f_{i k}$, link one ground-state level of ${ }^{133} \mathrm{Cs}(F=3$ or $F=4)$ to one of the $6 \mathrm{P}^{1 / 2}$ excited state levels $k\left(F^{\prime}=3\right.$ or $\left.F^{\prime}=4\right)$. The total light-shift $\delta v_{00}$ of the microwave atomic clock transition (in our case $\left|F=3, \quad m_{\mathrm{F}}=0>\rightarrow\right| F=4$, $m_{\mathrm{F}}=0>$ ) is the difference of the involved ground-state level shifts (including the contribution from the offresonant excited state by summation over $k$ in Eq. 1):

$\delta v_{00}\left(f_{\mathrm{L}}, I\right)=\delta v_{4}\left(f_{\mathrm{L}}, I\right)-\delta v_{3}\left(f_{\mathrm{L}}, I\right)$

This light-shift $\delta v_{00}$ of the clock transition is shown schematically in Fig. $2 \mathrm{~b}$, as a function of the frequency $f_{\mathrm{L}}$

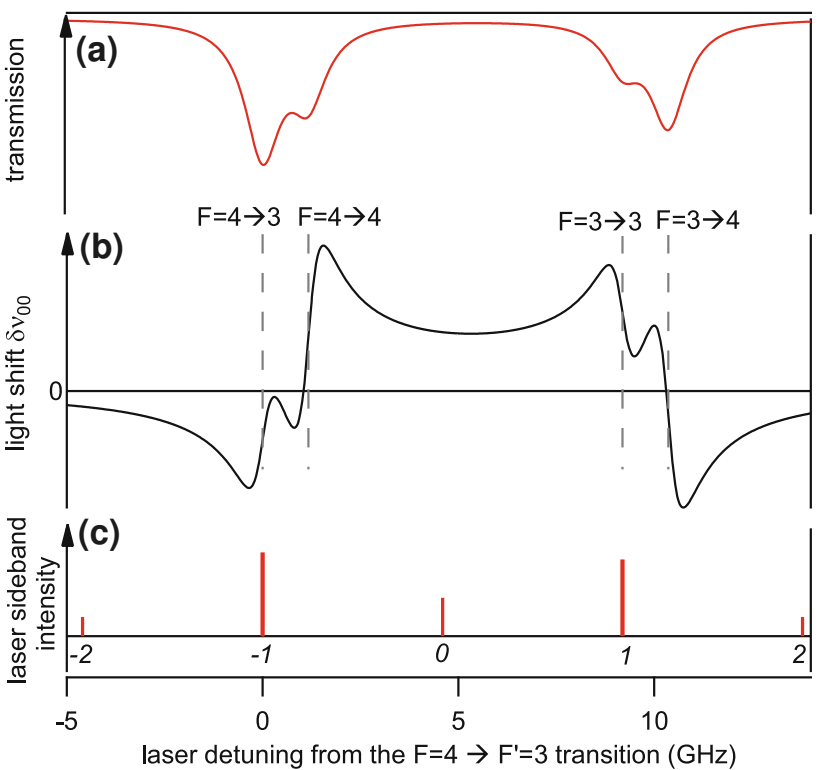

Fig. 2 Schematic view of light-shift in the case of CPT on the Cs D1 line. a Example of an optical transmission spectrum for a thermal Cs vapour, showing line broadening by a buffer-gas. b Frequencydependence of the light-shift $\delta v_{00}$ of the clock transition, for a monochromatic constant-intensity laser. The dashed vertical lines indicate the positions of the optical transitions. $\mathbf{c}$ Typical example of a multi-frequency laser spectrum, for CPT excitation by the positive and negative first-order sidebands. Italic numbers give the order $j$ of each sideband. The resulting clock transition light-shift under polychromatic excitation is the product of spectra $\mathbf{b}$ with $\mathbf{c}$, summed over all laser sidebands

for a monochromatic laser field varied around the Cs D1 transition. In the case of a multi-frequency laser spectrum, e.g. for CPT induced with a frequency-modulated laser, the total light-shift is obtained as the sum over the contributions from all existing laser frequency components $f_{j}$ with their respective intensities $I_{j}$ :

$\delta v_{00}\left(f_{\mathrm{L}}, I\right)=\sum_{j}\left[\delta v_{4}\left(f_{j}, I_{j}\right)-\delta v_{3}\left(f_{j}, I_{j}\right)\right]$

where $f_{\mathrm{L}}$ is the laser carrier frequency $(j=0)$ here and $j \neq 0$ numbers the modulation sidebands. A typical case of a multi-frequency laser spectrum used for CPT excitation is depicted in Fig. 2c.

In an atomic clock application, the light-shift can be expressed locally (i.e. at a given laser frequency and laser intensity) by coefficients known as the intensity light-shift coefficient $\alpha$ and the frequency light-shift coefficient $\beta$, by partial derivation of Eq. (2) or (3):

$\alpha\left(f_{\mathrm{L}}, I\right)=\frac{\mathrm{d} v_{00}}{\mathrm{~d} I} \quad$ and $\beta\left(f_{\mathrm{L}}, I\right)=\frac{\mathrm{d} v_{00}}{\mathrm{~d} f_{\mathrm{L}}}$

With $\alpha$ and $\beta$ having in general non-zero value, it is evident that residual fluctuations in the laser intensity or laser frequency give rise to fluctuations in the atomic level 
energies and thus to instabilities of the atomic clock. It is common practice to express the light-shift coefficients and clock frequency stability in fractional values, i.e. normalized to $v_{00}$.

Several methods have been developed for light-shift reduction in commercial lamp-pumped Rb clocks [19, 32], in laboratory clocks based on laser optical pumping [31, 33 and references therein, 34-35], and in CPT-based vapourcell clocks [27, 36-39]. In the case of a CPT-based clock with continuous-wave excitation-as considered here for a miniature atomic clock-the light-shift can be significantly reduced below the level encountered in optically pumped atomic clocks by optimizing the modulation index of the $\mathrm{RF}$ frequency-modulated laser field used to create the CPT signal $[27,36]$. However, the light-shift remains a critical issue for the atomic clock, and there are no methods reported for light-shift reduction in a CPT atomic clock using the Cs D1 line. In this communication, we report on suppression of the light-shift using the Cs D1 line for the case of a frequency-modulated laser field, but as a function of the Cs cell temperature.

\section{Experimental setup}

The block diagram of the experimental clock setup is shown in Fig. 3. The CPT resonance is prepared using a distributed feedback (DFB) laser emitting at $894.6 \mathrm{~nm}$ [40], i.e. resonant with the Cs D1-line. This choice allows for a CPT resonance with higher contrast and narrower linewidth compared to excitation on the D2-line [41]. Two phase-coherent electromagnetic fields, separated by $2 v \approx v_{\mathrm{Cs}} \approx 9.2 \mathrm{GHz}$, are generated by modulating the laser frequency at $v \approx 4.6 \mathrm{GHz}$ using a fibre-coupled electro-optical modulator (EOM). The two first-order sidebands are used to create the CPT effect. In our experiments $3.5 \mathrm{dBm}$ of $\mathrm{RF}$ power $\left(P_{\mathrm{RF}}\right)$ was applied, resulting in a phase-modulation index of $M=1.7$. This corresponds to 35(2) and 32(2) \% of the optical power transferred to the blue and red first-order sidebands, respectively; 16(2) \% is in the carrier while the remaining power is distributed in higher-order sidebands. The $4.6 \mathrm{GHz}$ frequency is generated by a commercial frequency synthesizer, referenced to a $10 \mathrm{MHz}$ quartz local oscillator. The quartz is compared to a hydrogen maser, monitored against a GPS (Global Positioning System) receiver for improved accuracy in our measurements. A beam splitter is placed directly after the EOM in order to split off one part of the optical beam and send it through a $25 \mathrm{~mm}$ long and $26 \mathrm{~mm}$ diameter glass-blown evacuated Cs cell. The resulting absorption signal can be used to stabilize the laser frequency using a standard lock-in scheme. The second part of the laser beam is circularly polarized by a quartz plate before passing through a MEMS Cs buffer-gas cell. This MEMS cell, realized using the process described in [42], is filled with $10 \mathrm{kPa}$ of $\mathrm{Ne}$ and has $2 \mathrm{~mm}$ internal diameter and $1.4 \mathrm{~mm}$ internal length. The MEMS cell is placed in a clock physics package (PP) where it is heated to a cell temperature $T_{\mathrm{C}}$ in the $45-90{ }^{\circ} \mathrm{C}$ range and temperaturecontrolled with an uncertainty $<0.1^{\circ} \mathrm{C}$. The PP also contains a solenoid which generates a static magnetic field of $12 \mu \mathrm{T}$ across the cell, oriented parallel to the light propagation vector to split the degeneracy of the Zeeman sublevels. Finally, two mu-metal magnetic shields complete the PP, to reduce perturbations from external magnetic fields. At the entrance to the MEMS cell, the laser beam has a Gaussian profile with diameter 2.08(2) $\mathrm{mm}$, centred with respect to the cell diameter.

A Si photodiode PD1 is placed directly after the physics package to detect the transmitted CPT signal. In most experimental situations, the laser frequency is stabilized using this signal from the MEMS cell, instead of the one from the evacuated cell (PD2). In this first case, the laser
Fig. 3 Block diagram of the experimental setup. $L A 1$ is a lock-in amplifier operating at $50 \mathrm{kHz}$, used for laser frequency stabilization. $L A 2$ is a second lock-in amplifier operating at $660 \mathrm{~Hz}$, used to increase signal-to-noise ratio of the clock signal. $P D 1$ is a photodetector detecting the CPT (clock) signal, $P D 2$ a photodetector detecting the saturated-absorption signal from the evacuated cell, and $P P$ the physics package. The solid black arrows indicate the optical paths and the dashed black arrows indicate the electrical paths

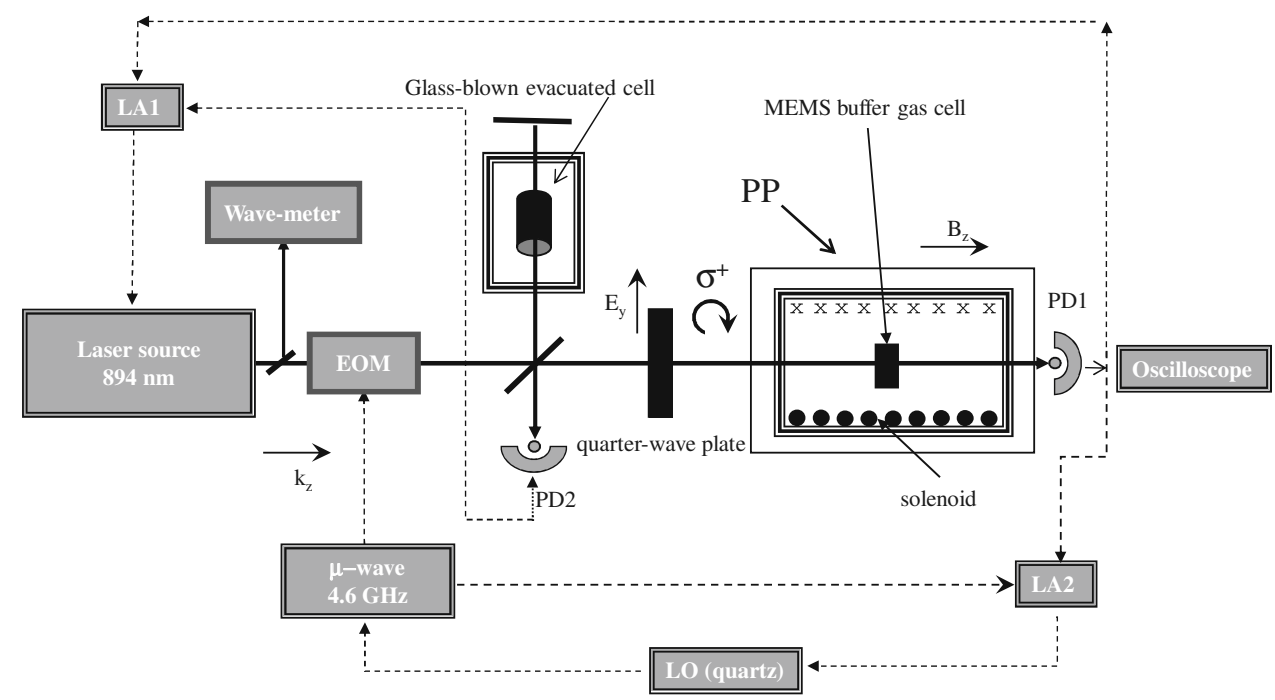


frequency is stabilized to a broadened line (Voigt profile resulting from Doppler and homogeneous broadening due to the buffer-gas inside the MEMS cell). About $10 \%$ of the laser beam intensity is split off directly after the laser and is directed to a high-resolution wavemeter used for precise laser frequency measurements. The wavemeter has a resolution of $10 \mathrm{MHz}$ and an accuracy of $30 \mathrm{MHz}$ and is calibrated using evacuated caesium and rubidium cells. In order to increase the signal-to-noise ratio, the frequency $v$ is frequency-modulated at $660 \mathrm{~Hz}$ and the CPT signal is detected with a second lock-in amplifier. The centre frequencies of the clock transition are obtained from fit functions to the lock-in resonance signals obtained by scanning $v$ through the CPT resonance condition.

A typical laser absorption signal for our excitation scheme is shown in Fig. 4a, observed on the MEMS cell: the dotted black line is the optical absorption spectrum
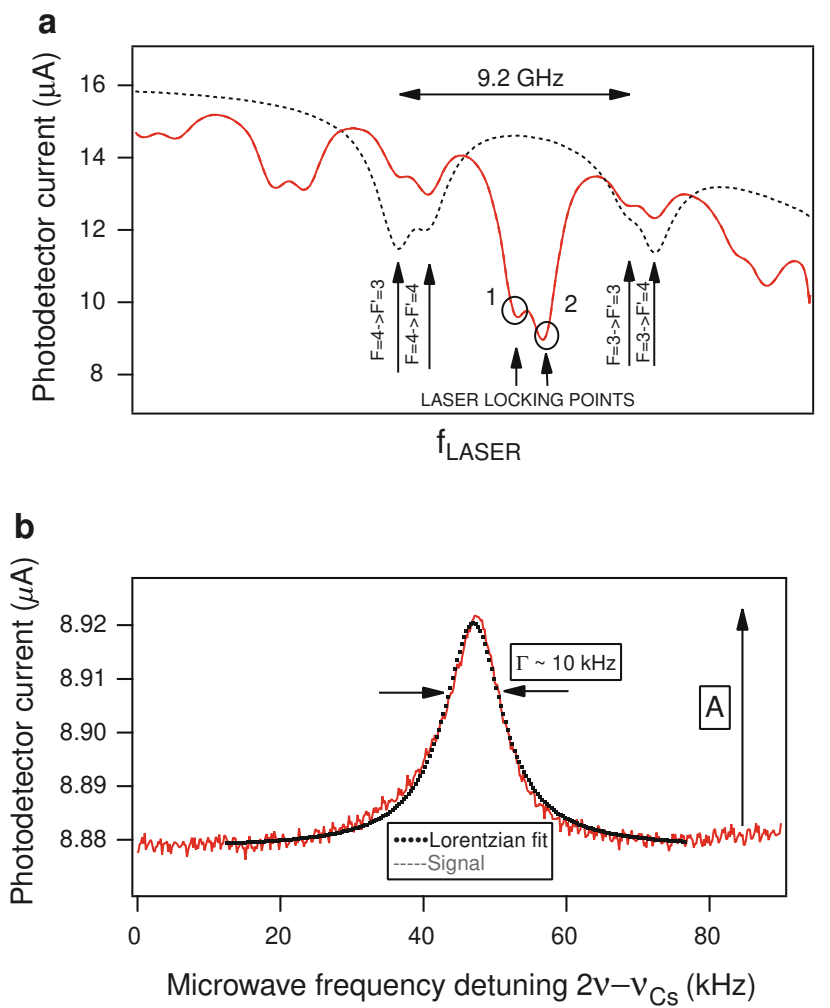

Fig. 4 a Dashed black line indicates absorption spectrum obtained with a single, non-modulated light field scanned over the Cs D1 line, for the MEMS cell heated to $T_{\mathrm{C}}=80.0(1){ }^{\circ} \mathrm{C}$. Resonant absorption of the laser light is 21(1) \% at the $F=4 \rightarrow F^{\prime}=3$ transition, in agreement with expectations from the Lambert-Beer law. Solid red line indicates same spectrum obtained with the modulated light field. CPT spectra are obtained with the laser carrier frequency stabilized to the laser locking points 1 or 2 , corresponding to coupling the ground states via the excited states $F^{\prime}=3$ or $F^{\prime}=4$, respectively, using the two first-order laser sidebands. b CPT signal observed on the MEMS cell heated to $T_{\mathrm{C}}=80.0{ }^{\circ} \mathrm{C}$. The signal amplitude is $A=0.042(2) \mu \mathrm{A}$, linewidth $\Gamma=10.1(1) \mathrm{kHz}$, and contrast $C=A / 8.88 \mu \mathrm{A}=0.5 \%$. Photodetector efficiency is $0.23 \mathrm{~A} / \mathrm{W}$ recorded by sweeping a single, non-modulated laser frequency through the Cs D1-line. The solid red line shows the same spectrum obtained with the laser frequencymodulated at $v=4.6 \mathrm{GHz}$. The Ne buffer-gas content in the MEMS cells contributes a homogeneous broadening of the optical transitions by $815 \mathrm{MHz}$ and also shifts the absorption lines by $-120 \mathrm{MHz}$ compared to the evacuated Cs cell [43]. An example of a CPT resonance is reported in Fig. 4b.

\section{Light-shift studies}

Figure 5 shows the shift of the clock transition frequency $v_{00}$ as function of the total laser intensity, for four different RF power levels driving the EOM (i.e. different modulation indices $M$ ). CPT coupling is via the $F^{\prime}=3$ excited state (laser carrier frequency stabilized to point 1 in Fig. 4a).

We find indeed that the intensity light-shift coefficient $\alpha$ (slope of the data in Fig. 5) can be minimized by proper choice of the modulation index $M$ (via the RF modulation power $P_{\mathrm{RF}}$ here) [36]. For $P_{\mathrm{RF}}=3 \mathrm{dBm}$, we find $\alpha=1.6 \times 10^{-12} \mathrm{~cm}^{2} / \mu \mathrm{W}$ while for $P_{\mathrm{RF}}=2.5$ and $3.5 \mathrm{dBm} \alpha$ is increased by factors of +4.5 and -4.5 , respectively. Such light-shift reduction has already been demonstrated on the Cs D2 line using a current-modulated extended-cavity diode laser (ECDL) [27] and currentmodulated vertical-cavity surface-emitted lasers (VCSELs) [39]. The same principle was also demonstrated on the $\mathrm{Rb}$ D1 line using current-modulated VCSELs [37, 38, 44].

In the following, we will show that a reduction of the intensity light-shift $\alpha$ can also be achieved by adjusting the temperature of the MEMS cell (instead of the modulation index), using the case of $P_{\mathrm{RF}}=3.5 \mathrm{dBm}$ of Fig. 4, for which $\alpha$ is not strongly suppressed. For this value of

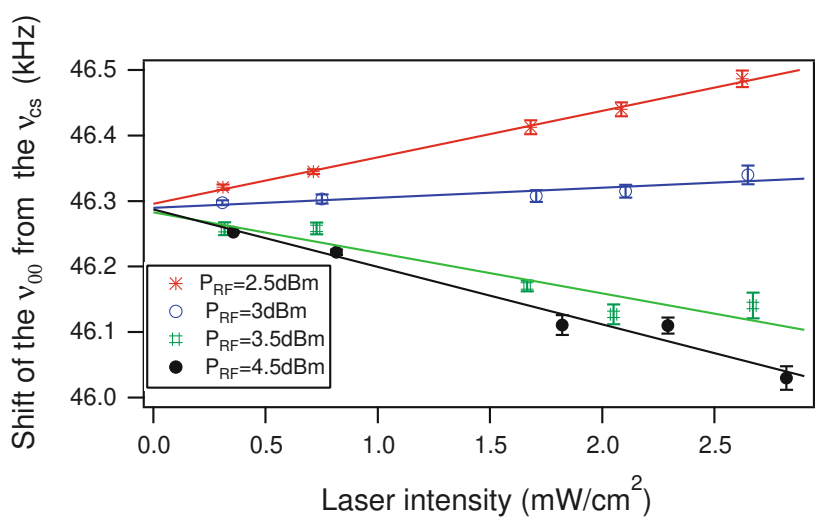

Fig. 5 Shift of the CPT clock transition as function of total light intensity, for different RF powers $P_{\mathrm{RF}}$. MEMS cell temperature is $T_{\mathrm{C}}=80^{\circ} \mathrm{C}$. The lines are linear fits to the data 

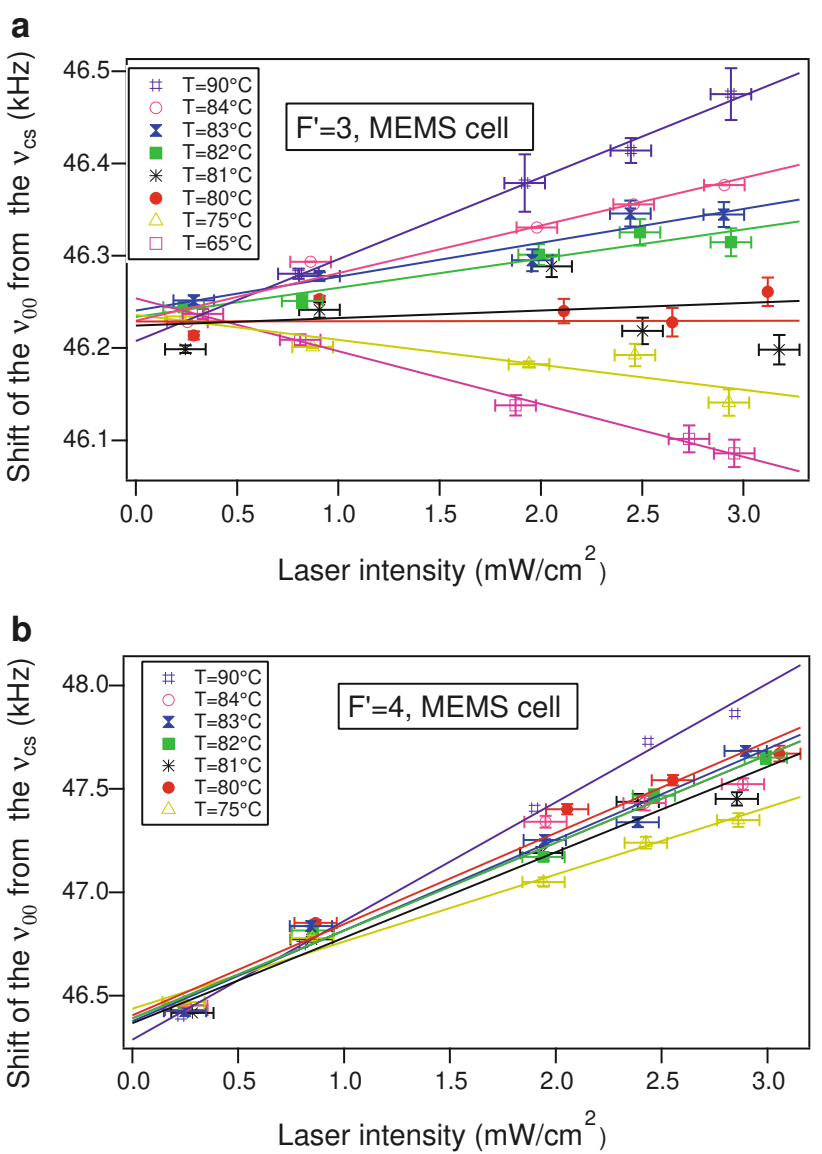

Fig. 6 Shift of the CPT clock transition measured on the MEMS cell, as function of laser intensity for different cell temperatures. a Results for the laser frequency tuned for coupling via the $F^{\prime}=3$ excited state. b Corresponding data for the laser frequency tuned for coupling via the $F^{\prime}=4$ state. Solid lines are linear fits to the data. Note the different ranges for $v_{00}$ in the two graphs

$P_{\mathrm{RF}}$, we obtained higher CPT signal amplitudes than for $P_{\mathrm{RF}}=3.0 \mathrm{dBm}$ where $\alpha$ is suppressed.

Figure 6 shows the measured shift of the CPT clock transition as a function of laser intensity, for different MEMS cell temperatures. The RF power is $P_{\mathrm{RF}}=3.5$ $\mathrm{dBm}$, and the measurements shown in Fig. $6 \mathrm{a}, \mathrm{b}$ are performed for coupling via the $F^{\prime}=3$ and $F^{\prime}=4$ excited states, respectively (laser locking points 1 and 2, respectively, in Fig. 4a).

When coupling via the $F^{\prime}=3$ excited state (Fig. 6a), the intensity light-shift coefficient $\alpha$ (given by the slope of the lines) can be adjusted over a wide range around zero value by changing the cell temperature: $\alpha$ is strongly suppressed at $T_{\mathrm{C}}=81^{\circ} \mathrm{C}$, while for lower temperatures $\alpha$ is negative and for higher temperatures $\alpha$ is positive. In the case of coupling via $F^{\prime}=4$ (Fig. $6 \mathrm{~b}$ ), $\alpha$ is always positive for any cell temperature used and we do not find a cell temperature with similarly strong suppression of $\alpha$ as for coupling via $F^{\prime}=3$. Similar results are obtained when the laser frequency is stabilized to the same transitions in the evacuated Cs cell; in

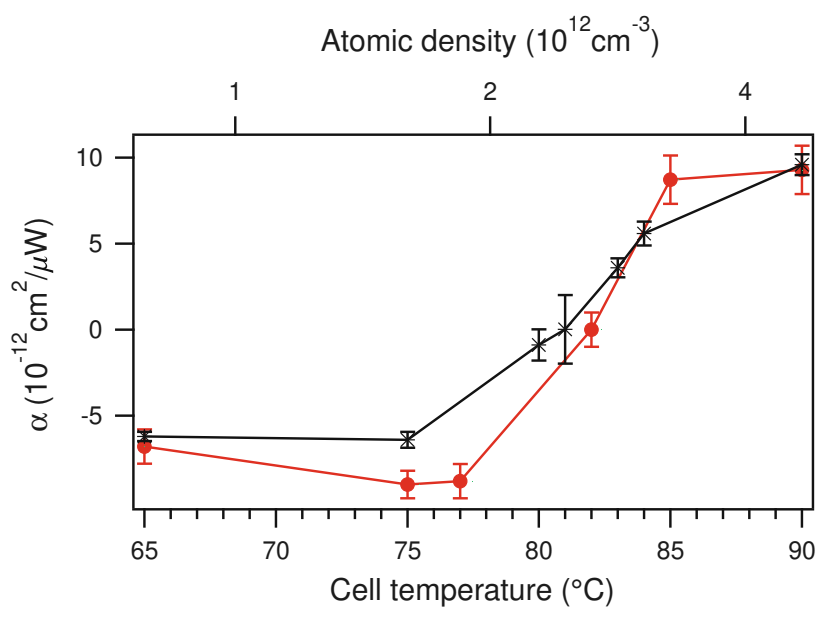

Fig. 7 Intensity light-shift coefficient $\alpha$ as a function of the cell temperature (red circles). The measurement was repeated after 14 months (black stars). $\alpha$ crosses the zero value level for a cell temperature of $81{ }^{\circ} \mathrm{C}$. Coupling is via the $F^{\prime}=3$ excited state (laser locking point 1 in Fig. 4a)

this case the effective laser detuning acting on the atoms in the MEMS cell is changed by the buffer-gas shift of $120 \mathrm{MHz}$. Note that a similar effect was reported for the case of an optically pumped double-resonance ${ }^{87} \mathrm{Rb}$ clock [45] where the intensity light-shift coefficient $\alpha$ is significantly suppressed for coupling to the higher-lying excited state $\left(F^{\prime}=2\right)$, but not for CPT clocks or Cs.

Numerical values for $\alpha$ were extracted from the slopes of the linear fits in Fig. 6a, and are shown in Fig. 7 as a function of the cell temperature. It is clearly seen that $\alpha$ crosses the zero value level for $T_{(\alpha=0)}=81.0(7){ }^{\circ} \mathrm{C}$ (red dots), which is reconfirmed by a second series of measurements performed 14 months later (black stars, $\left.T_{(\alpha=0)}=80.3(3){ }^{\circ} \mathrm{C}\right)$. Both datasets show the same general behaviour of $\alpha$ with temperature, but some differences are visible. There is some experimental evidence that for $T_{\mathrm{C}}<90{ }^{\circ} \mathrm{C}$ Cs droplets form on the cell windows and can block a part of the light intensity incident on the cell, which might explain the observed differences between the two datasets. Between the two datasets, we also find a frequency drift of the clock transition $v_{00}$ of $-6.3 \times 10^{-9} /$ month.

The shift of the clock transition $v_{00}$ as a function of the laser frequency is shown in Fig. 8a, for different laser intensities and a MEMS cell temperature of $T_{\mathrm{C}}=65^{\circ} \mathrm{C}$. These data are recorded for the laser frequency varied slightly around $335.1160(3) \mathrm{THz}$, i.e. for coupling via the $F^{\prime}=3$ excited state (laser frequency close to point 1 in Fig. 4a). The frequency light-shift coefficient $\beta$ is given by the slopes of the line fits in Fig. 8a, e.g. $\beta=-644 \mathrm{mHz} /$ $\mathrm{MHz}$ for a total laser intensity of $4.04 \mathrm{~mW} / \mathrm{cm}^{2}$. The intersection of the three line fits (for different light intensities) gives the laser frequency for which a zero $\alpha$-coefficient is obtained. This intersection point depends 

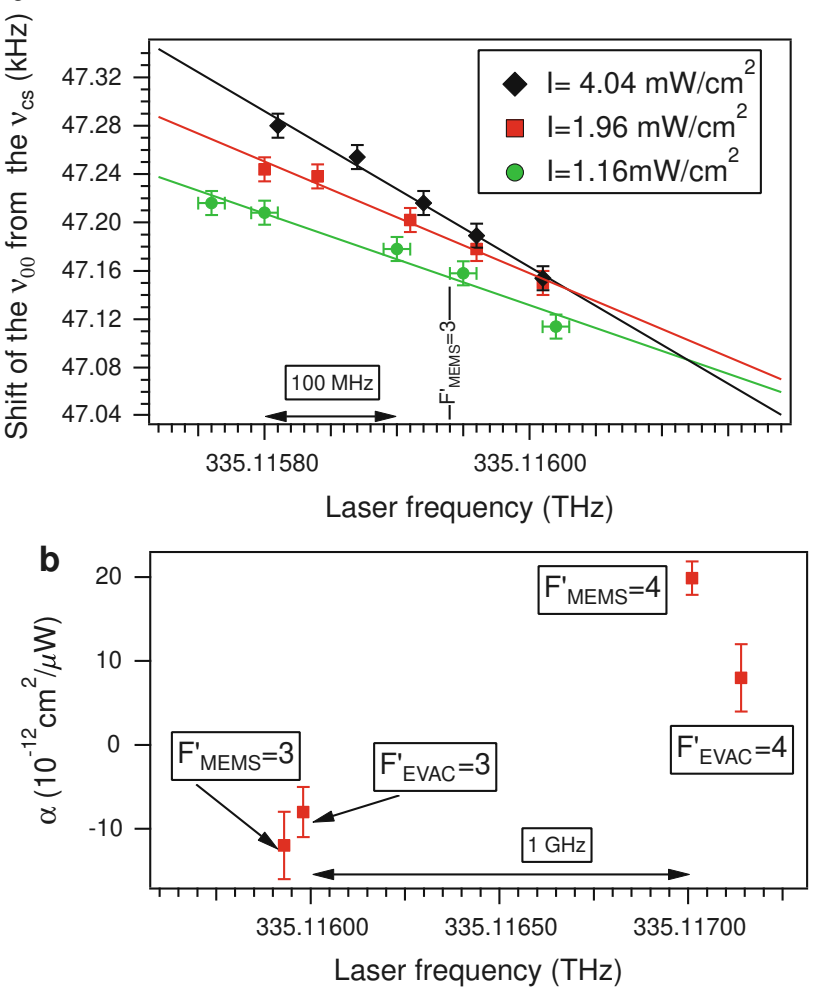

Fig. 8 a Shift of the clock resonance line as a function of laser frequency, measured for different total laser intensities (I). The lightshift coefficient $\beta$ is given by the slopes of the line fits. b Intensity light-shift coefficient $\alpha$, for laser frequencies stabilized to the $F \rightarrow F^{\prime}=3$ or $F \rightarrow F^{\prime}=4$ transitions in either the MEMS cell or the evacuated cell. The MEMS cell temperature is $65^{\circ} \mathrm{C}$ for both graphs. In the MEMS cell, the optical transitions have a linewidth of $1.1 \mathrm{GHz}(\mathrm{FWHM})$ and are shifted by $-120 \mathrm{MHz}$ with respect to those in the evacuated Cs cell

on the cell temperature, and for $T_{\mathrm{C}}=65{ }^{\circ} \mathrm{C}$ this point is at $335.11603(3) \mathrm{THz}$, which is closer to the transition $F \rightarrow F^{\prime}=3$ in the evacuated cell than to the same transition in the MEMS cell. (For a cell temperature of $T_{\mathrm{C}}=81{ }^{\circ} \mathrm{C}$ this intersection point is extrapolated to be at 335.11628(3) THz.)

This latter finding is confirmed by the data of Fig. 8b, where the intensity light-shift coefficient $\alpha$ is shown for the laser frequency stabilized to the four possible laser lock points, namely for coupling via the $F^{\prime}=3$ or $F^{\prime}=4$ excited states in either the MEMS cell or the evacuated cell. The smallest intensity light-shift (lowest value of $|\alpha|$ ) is found with the laser stabilized to the $F \rightarrow F^{\prime}=3$ transitions in the evacuated cell, close to 335.1160(3) THz.

\section{Discussion of the light-shift studies}

Our experimental results of Fig. 6a show that the intensity light-shift coefficient $\alpha$ can change its sign or be brought close to zero value depending on the cell temperature, when coupling via the $F^{\prime}=3$ excited state. We propose a qualitative explanation of this effect, based on a change in overall balance between positive and negative light-shift contributions from the different laser sidebands, induced by the change in cell temperature. From Fig. 2 it can be seen that for coupling via the $F^{\prime}=3$ excited state, the carrier and +1 st-order sideband contribute a positive light-shift, while all other sidebands contribute a negative light-shift. Our experiments show, that at low cell temperature $T_{\mathrm{C}}<80^{\circ} \mathrm{C}$, the overall light-shift is negative (see Fig. 7). With increasing cell temperature, the atomic density in the cell increases and hence the resonant light (both first-order sidebands) is absorbed more strongly in the cell: we measure the combined absorption of the two first-order sidebands (at point 1 in Fig. 4a) to go from $\mathrm{I}_{\text {absorbed }} / I_{\text {incident }}=$ $10(1) \%$ at $T_{\mathrm{C}}=65{ }^{\circ} \mathrm{C}$ to $53(1) \%$ at $T_{\mathrm{C}}=90{ }^{\circ} \mathrm{C}$, while the carrier and higher-order sidebands are transmitted essentially non-attenuated through the cell. Because the measured light-shift depends on the average optical intensity (spatial average along the light propagation direction over the cell), the temperature-induced increase in optical absorption on the $F=4 \rightarrow F^{\prime}=3$ transition will reduce the negative light-shift contribution from the negative 1st-order sideband more strongly than the smaller increase of optical absorption on the $F=3 \rightarrow F^{\prime}=3$ transition reduces the positive light-shift contribution from the positive 1st-order sideband. As a result, the overall intensity light-shift from all different laser sidebands changes from negative to positive, as it is seen in our measurements. Another mechanism potentially contributing to the observed effect could be a frequency shift of the frequency-stabilized laser with changing cell temperature: due to the strong collisional broadening in the MEMS cell (Fig. 4a, points 1 and 2), the wing of the Doppler absorption line from the off-resonant excited state can introduce a temperature-dependent offset in the lock-in error signal and therefore shift the laser frequency. However, our measurements of the laser frequency do not confirm such shifts within the wavemeter resolution of $10 \mathrm{MHz}$, which corresponds to a negligible $<6 \mathrm{~Hz}$ shift of $v_{00}$ in our experiments.

When coupling via the $F^{\prime}=4$ excited state, the intensity light-shift coefficient $\alpha$ is always positive (Fig. 6b). Here the initial absolute value of $\alpha$ is too large (see also Fig. 8b) compared to the adjustment range of $\delta \alpha \approx 1.5 \times 10^{-11}$ $\mathrm{cm}^{2} / \mu \mathrm{W}$ achievable by changing the cell temperature, which is on the same order for both couplings via $F^{\prime}=3$ and $F^{\prime}=4$ (see Fig. 6a, b). This prevents obtaining a suppressed $\alpha$ value when coupling via $F^{\prime}=4$ in the same range of cell temperatures, and we observe positive $\alpha$ only.

Our results show that conditions exist where the intensity light-shift coefficient $\alpha$ can be minimized by choosing an appropriate cell temperature, even in the case of non- 
Table 1 Comparison of the different intensity light-shift coefficients $\alpha$ and frequency light-shift coefficients $\beta$ reported in the literature and in this work

\begin{tabular}{|c|c|c|c|c|c|}
\hline Reference & Nagel et al. [46] & Knappe et al. [28] & Lutwak et al. [48] & Boudot et al. [39] & This work \\
\hline Transition & Cs $\mathrm{D}_{2}$ & Cs $\mathrm{D}_{2}$ & Cs $\mathrm{D}_{2}$ & Cs $\mathrm{D}_{2}$ & Cs $D_{1}$ \\
\hline Buffer-gas & $\mathrm{Ne}$ & $\mathrm{Ne}$ & $\mathrm{N}_{2} / \mathrm{Ar}$ & $\mathrm{Ne}$ & $\mathrm{Ne}$ \\
\hline Laser & Two phase-locked lasers & VCSEL & VCSEL & VCSEL & $\mathrm{DFB}+\mathrm{EOM}$ \\
\hline$I_{-1} / I_{0} / I_{+1}(\%)$ & $60 / 0 / 40$ & $19 / 8 / 47$ & No data & $14 / 14 / 33$ & $32 / 16 / 35$ \\
\hline$\alpha\left(\mu \mathrm{W} / \mathrm{cm}^{2}\right)^{-1}$ & $8.7 \times 10^{-10}$ & $1.08 \times 10^{-10}$ & $1.6 \times 10^{-10}$ & $-3.2 \times 10^{-12}$ & $-1.7 \times 10^{-13}$ \\
\hline$\beta(\mathrm{mHz} / \mathrm{MHz})$ & No data & -20 & No data & No data & -11 \\
\hline
\end{tabular}

The values for $\beta$ are normalized to the resonant laser intensity of $I=45 \mu \mathrm{W} / \mathrm{cm}^{2}$ used in [28]. The line labelled " $\mathrm{I}_{-1} / \mathrm{I}_{0} / \mathrm{I}_{+1}$ " gives the percentage of total light intensity contained in the -1 st-order sideband, the carrier, and the +1 st-order sideband in the respective studies (CPT is induced using the two first-order sidebands in all cases)

optimized phase-modulation index $M$. This allows working with a higher CPT signal amplitude for improved clock stability (as is the case for our data obtained with $P_{\mathrm{RF}}=3.5 \mathrm{dBm}$ ) compared to the light-shift compensation scheme using adjustment of $M$ only, or to adjust $M$ for minimized frequency light-shift $\beta$.

In the literature, no frequency and intensity light-shift coefficients are reported for a CPT atomic clock using the Cs D1 line. The previously published coefficients are obtained on the Cs D2 line and are shown in Table 1, along with our data as reported above for laser stabilization to the $F \rightarrow F^{\prime}=3$ transitions in the MEMS cell. In particular, we find that our result for $\beta$ reported in Sect. 4 above has the same sign, but is a factor of 2 smaller than the value reported in [28], when rescaled to the resonant laser intensity used in [28]. This difference can be attributed to the different intensities of the first-order sidebands in both works (see Table 1), resulting in a different degree of $\beta$ compensation between these sidebands (see Fig. 2b, c). In our experiments, the first-order laser sidebands are of almost equal intensity, while in [28] a current-modulated VCSEL is used and amplitude modulation causes a stronger sideband asymmetry (the Ne pressures used in these works differ by $3 \%$ only). It has been demonstrated that the intensity light-shift $\alpha$ is inversely proportional to the buffer-gas pressure, essentially due to the broadening of the optical resonance [46], so further reduction of the light-shift coefficients may be expected when using higher buffer-gas pressures [47].

\section{Impact of light-shift and temperature coefficient on the clock stability}

In addition to the light-shift, the temperature coefficient of the clock cell is another main source of instability in atomic clocks. Our light-shift studies allow us to isolate the temperature coefficient of the MEMS Cs cell with $\mathrm{Ne}$ buffer-gas content, by extrapolating the CPT clock frequency $v_{00}$ to zero light intensity. In general, the presence of a buffer-gas in alkali cells prevents relaxation due to atom-wall collisions which otherwise would strongly broaden the resonance linewidth. With each buffer-gas species (typically $\mathrm{Ar}, \mathrm{Ne}, \mathrm{N}_{2}$, etc.) is associated a shift of clock frequency which depends on the gas pressure and temperature (temperature coefficient) and thus links the clock frequency to the environmental conditions. To limit this effect, a mixture of two buffer-gases with opposite temperature coefficients is generally used, to obtain a suppressed linear temperature coefficient at a specific inversion temperature [49-51]. We previously demonstrated that an inversion temperature at $T_{\mathrm{C}}=80^{\circ} \mathrm{C}$ can also be obtained with pure Ne as buffer-gas [52], without need for a gas mixture. This temperature coincides with the cell temperature for suppressed intensity light-shift reported above, for coupling via the $F^{\prime}=3$ excited state, and it is also in the range of suitable temperatures for clock operation.

The short-term frequency stability of an atomic clock, described by the Allan deviation $\sigma_{y}(\tau)$, can be estimated by the detection noise (noisePSD) and the resonance discriminator slope $(D)$, following Eq. (5):

$\sigma_{y}(\tau)=\frac{\text { noisePSD }}{\sqrt{2} D v} \times \tau^{-1 / 2}$

Here, $v$ is the microwave modulation frequency and $D$ is the discriminator slope, determined by the CPT linewidth, amplitude, and the demodulation scheme implemented for signal detection.

To measure the clock stability, the $4.6 \mathrm{GHz}$ microwave radiation produced by the microwave synthesizer is stabilized to the centre of the CPT line. This is achieved by steering the quartz local oscillator's frequency of $\sim 10 \mathrm{MHz}$ using a feedback loop based on the output from lock-in amplifier LA2 (see Fig. 3). The $10 \mathrm{MHz}$ output of the quartz oscillator is then compared to the signal of a hydrogen maser using a frequency comparator. The measured clock stabilities are reported in Fig. 9. The squares represent the clock stability for measurements before we 


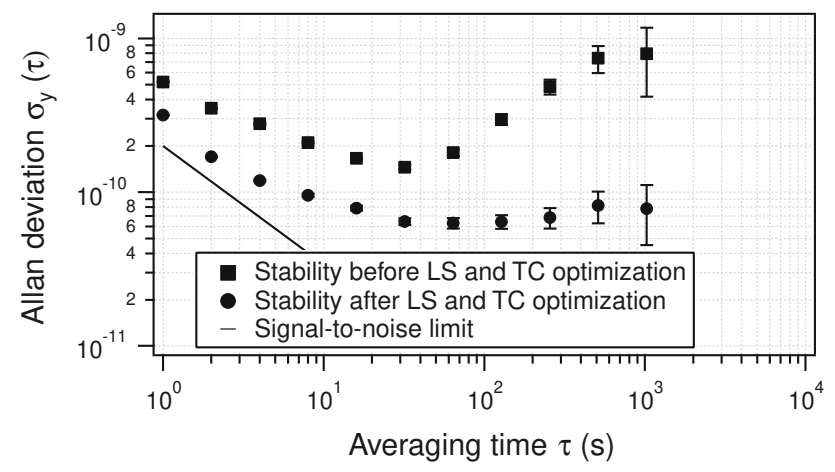

Fig. 9 Short-term clock frequency stability measurement, measured before (squares) and after (circles) setting the clock parameters for suppressed light-shift and temperature coefficients

optimized the system for suppressed light-shift and temperature coefficients. The circles give the clock frequency stability after setting the parameters as reported above, in order to have low intensity light-shift and temperature coefficient $\left(T_{\mathrm{C}}=80^{\circ} \mathrm{C}, P_{\mathrm{RF}}=3.5 \mathrm{dBm}\right)$. At mediumterm timescales $(\approx 1,000 \mathrm{~s})$ an improvement in stability by one order of magnitude is seen, due to optimized light-shift and temperature coefficients. With a laser frequency stability on the level of $1 \mathrm{MHz}$ [53], the frequency light-shift contributes to the clock frequency stability on the level of $7 \times 10^{-11}$ around $\tau=1,000 \mathrm{~s}$, which is the dominating contribution here. The intensity light-shift gives an instability contribution on the level of $10^{-13}$ (estimated relative instability of the laser intensity is $0.1 \%$ ), while the cell's temperature coefficient contributes $<2 \times 10^{-13}$ for a cell temperature stability on the $10 \mathrm{mK}$ level [52].

\section{Conclusions}

We presented experimental results on the light-shift in a MEMS cell filled with Cs and pure Ne buffer-gas, for CPT spectroscopy on the Cs D1 line (at $894.6 \mathrm{~nm}$ ). The behaviour of the light-shift of the CPT clock transition was studied, as a function of laser frequency and intensity, and for different cell temperatures. We found in particular that the intensity light-shift coefficient $\alpha$ depends on the cell temperature, and that the cell temperature can be set to have $\alpha$ significantly suppressed, at approximately the same temperature at which a suppressed linear temperature coefficient occurs in the Ne-filled MEMS Cs cell. This approach has the advantage that the laser's modulation index $M$ can still be adjusted for minimized frequency light-shift $\beta$ or for optimized short-term clock stability, which is more difficult in light-shift suppression techniques based on adjustment of $M$ only. Under optimized conditions, the measured clock stability is improved by one order of magnitude to $\sigma_{y}(\tau)<1 \times 10^{-10}$ at $\tau=100-1,000 \mathrm{~s}$, mainly limited by the frequency lightshift at these integration times. These results are of high relevance for the realization of novel miniature atomic clocks based on CPT on the Cs D1 line. The demonstrated method is also expected to be applicable to other cell dimensions and other alkali atoms.

Note added in proof: after submission of the manuscript we became aware of similar unpublished results on the dependence of $\alpha$ on cell temperature, obtained by the team at SYRTE for a pulsed CPT Cs cell clock (S. Guérandel and J.-M. Danet, private communication). However, their temperatures for minimized light-shift and temperature coefficient do not coincide.

Acknowledgments This work was funded by EU FP7 (MAC-TFC project, no. 224132) and by the Swiss National Science Foundation (project 200020-118062). We thank F. Gruet, P. Scherler, M. Durrenberger (all LTF-UniNe) for their contributions. We also thank V. Giordano and the team from FEMTO-ST CNRS-UFC, Besançon, France for helpful discussions, and $\mathrm{H}$. Schweda for a careful reading of the manuscript.

\section{References}

1. G. Alzetta, A. Gozzini, M. Moi, G. Orriols, Nuovo Cimento B 36, 5 (1976)

2. W.E. Bell, A.L. Bloom, Phys. Rev. Lett. 6, 280 (1961)

3. E. Arimondo, G. Orriols, Lett. Nuovo Cimento 17, 333 (1976)

4. H.R. Gray, R.M. Whitley, C.R. Stroud Jr., Opt. Lett. 3, 218 (1978)

5. F. Levi, A. Godone, C. Novero, J. Vanier, in Proceedings of the 11th European Frequency and Time Forum, 216 (1997)

6. J. Vanier, A. Godone, F. Levi, Phys. Rev. A 58, 2345 (1998)

7. N. Cyr, M. Tetu, M. Breton, IEEE Trans. Instrum. Meas. 42, 640 (1993)

8. S.E. Harris, J.E. Field, A. Imamoglu, Phys. Rev. Lett. 64, 1107 (1990)

9. A. Kasapi, M. Jain, G.Y. Yin, S.E. Harris, Phys. Rev. Lett. 74, 2447 (1995)

10. S.E. Harris, Phys. Today 50, 36 (1997)

11. A. Nagel, L. Graf, A. Naumov, E. Mariotti, V. Biancalana, D. Meschede, R. Wynands, Europhys. Lett. 44, 31 (1998)

12. A.L. Bloom, Appl. Opt. 1, 61 (1962)

13. M.O. Scully, M. Fleischhauer, Phys. Rev. Lett. 69, 1360 (1992)

14. A. Aspect, E. Arimondo, R. Kaizer, N. Vansteenkiste, C. CohenTannoudji, J. Opt. Soc. Am. B 6, 2112 (1989)

15. O. Schmidt, R. Wynands, Z. Hussein, D. Meschede, Phys. Rev. A 53, 27 (1996)

16. M. Bajcsy, A.S. Zibrov, M.D. Lukin, Nature 426, 638 (2003)

17. S. Brandt, A. Nagel, R. Wynands, D. Meschede, Phys. Rev. A 56, 1063 (1997)

18. M. Erhard, S. Nußmann, H. Helm, Phys. Rev. A 62, 061802 (2000)

19. J. Vanier, C. Audoin, The Quantum Physics of Atomic Frequency Standards (Adam Hilger, Bristol, 1989)

20. A. Godone, F. Levi, J. Vanier, Phys. Rev. A 59, 12 (1999)

21. M. Merimaa, T. Lindvall, I. Tittonen, E. Ikonen, J. Opt. Soc. Am. B 20, 273 (2003)

22. J. Vanier, Appl. Phys. B 81, 421 (2005)

23. R. Lutwak, D. Emmons, W. Riley, R.M. Garvey, in Proceedings of the 34th Annual Precise Time and Time Interval (PTTI) Meeting, 539 (2002) 
24. J. Vanier, M.W. Levine, D. Janssen, M.J. Delaney, IEEE Trans. Instrum. Meas. 52, 822 (2003)

25. S. Knappe, V. Shah, P.D. Schwindt, L. Hollberg, J. Kitching, L.A. Liew, J. Moreland, Appl. Phys. Lett. 85, 1460 (2004)

26. R. Lutwak, D. Emmons, T. English, W. Riley, A. Duwel, M. Varghese, D. K. Serkland, G. M. Peake, in Proceedings of the 35th Precise Time and Time Interval (PTTI) Systems and Applications Meeting, 1 (2003)

27. F. Levi, A. Godone, J. Vanier, IEEE Trans. Ultrason. Ferroelectr. Freq. Control 47, 466 (2000)

28. S. Knappe, R. Wynands, J. Kitching, H.G. Robinson, L. Hollberg, J. Opt. Soc. Am. B 18, 1545 (2001)

29. C. Cohen-Tannoudji, J. Dupont-Roc, Phys. Rev. A 5, 968 (1972)

30. J. Barrat, C. Cohen-Tannoudji, J. Phys. Radium 22, 329 (1961)

31. J. Deng, IEEE Trans. Ultrason. Ferroelectr. Freq. Control 48, 1657 (2001)

32. J.C. Camparo, R.P. Frueholz, C.H. Volk, Phys. Rev. A 27, 1914 (1983)

33. C. Affolderbach, C. Andreeva, S. Cartaleva, T. Karaulanov, G. Mileti, D. Slavov, Appl. Phys. B 80, 841 (2005)

34. F. Levi, C. Novero, A. Godone, G. Brida, IEEE Trans. Instrum. Meas. 46, 128 (1997)

35. B.H. McGuyer, Y-.Y. Jau, W. Happer, Appl. Phys. Lett. 94, 251110 (2009)

36. M. Zhu, L. S. Cutler, in Proceedings of the 32nd Precise Time and Time Interval Systems and Applications Meeting, ed. by L.A. Breakiron (US Naval Observatory, Washington, DC, 2000), p. 311

37. V. Georginov, S. Knappe, P.D. Schwindt, L. Hollberg, J. Kitching, J. Opt. Soc. Am. B 23, 593 (2006)

38. E.E. Mikhailov, T. Horrom, N. Belcher, I. Novikova, J. Opt. Soc. Am. B 27, 417 (2010)

39. R. Boudot, P. Dziuban, M. Hasegawa, R.K. Chutani, S. Galliou, V. Giordano, C. Gorecki, J. Appl. Phys. 109, 014912 (2011)
40. F. Gruet, D. Miletic, C. Affolderbach, G. Mileti, V. Vilokkinen, P. Melanen, in Proceedings of the International Symposium on Reliability of Optoelectronics for Space, 1 (2009)

41. M. Stähler, R. Wynand, S. Knappe, J. Kitching, L. Hollberg, A. Taichenachev, V. Yudin, Opt. Lett. 27, 1472 (2002)

42. M. Hasegawa, R.K. Chutani, C. Gorecki, R. Boudot, P. Dziuban, V. Giordano, S. Clatot, L. Mauri, Actuators A: Phys. 167, 594 (2011)

43. G.A. Pitz, D.E. Wertepny, G.P. Perram, Phys. Rev. A 80, 062718 (2009)

44. V. Shah, V. Gerginov, P.D.D. Schwindt, S. Knappe, L. Hollberg, J. Kitching, Appl. Phys. Lett. 89, 151124 (2006)

45. J.Q. Deng, G. Mileti, J.M. Lopez-Romero, D.A. Jennings, F.L. Walls, R.E. Drullinger, in Proceedings of the 11th European Frequency and Time Forum, 211 (1997)

46. A. Nagel, S. Brandt, D. Meschede, R. Wynands, Europhys. Lett. 48, 385 (1999)

47. J. Camparo, J. Coffer, J. Townsend, J. Opt. Soc. Am. B 22, 521 (2005)

48. R. Lutwak, D. Emmons, W. Riley, R. M. Garvey, in Proceedings of the 34th Precise Time and Time Interval Systems and Applications Meeting, 1 (2002)

49. R. Boudot, D. Miletic, P. Dziuban, C. Affolderbach, P. Knapkiewicz, J. Dziuban, G. Mileti, V. Giordano, C. Gorecki, Opt. Express 19, 3106 (2011)

50. K. Deng, X. Chen, Z. Wang, Opt. Lett. 36, 1740 (2011)

51. O. Kozlova, S. Guérandel, E. de Clercq, Phys. Rev. A 83, 062714 (2011)

52. D. Miletic, P. Dziuban, R. Boudot, M. Hasegawa, R.K. Chutani, G. Mileti, V. Giordano, C. Gorecki, Electron. Lett. 46, 1069 (2010)

53. J. Di Francesco, F. Gruet, C. Schori, C. Affolderbach, R. Matthey, G. Mileti, Y. Salvadé, Y. Petremand, N. De Rooij, SPIE Proc. 7720, 7720IT-1 (2010) 